\title{
The vagus afferent network: emerging role in translational connectomics
}

\author{
Laureen D. Hachem, MD, ${ }^{1}$ Simeon M. Wong, BASc, ${ }^{2}$ and George M. Ibrahim, MD, PhD1,3 \\ ${ }^{1}$ Division of Neurosurgery, Department of Surgery, University of Toronto; ${ }^{2}$ Department of Diagnostic Imaging, Hospital for Sick \\ Children, Toronto; and ${ }^{3}$ Division of Neurosurgery, Hospital for Sick Children, Program in Neuroscience and Mental Health, \\ Hospital for Sick Children Research Institute, Toronto, Ontario, Canada
}

\begin{abstract}
Vagus nerve stimulation (VNS) is increasingly considered for the treatment of intractable epilepsy and holds potential for the management of a variety of neuropsychiatric conditions. The emergence of the field of connectomics and the introduction of large-scale modeling of neural networks has helped elucidate the underlying neurobiology of VNS, which may be variably expressed in patient populations and related to responsiveness to stimulation. In this report, the authors outline current data on the underlying neural circuitry believed to be implicated in VNS responsiveness in what the authors term the "vagus afferent network." The emerging role of biomarkers to predict treatment effect is further discussed and important avenues for future work are highlighted.
\end{abstract}

https://thejns.org/doi/abs/10.3171/2018.6.FOCUS18216

KEYWORDS epilepsy; neuromodulation; VNS; vagus nerve stimulation

$\mathrm{E}$ PILEPSY is among the most common serious neurological conditions affecting children worldwide. ${ }^{66}$ Despite advances in pharmacological treatments, approximately $30 \%$ of children develop medically intractable epilepsy requiring adjunctive therapy. ${ }^{40}$ Vagus nerve stimulation (VNS) has emerged as a promising treatment modality in the management of medically intractable epilepsy to reduce seizure rates. Despite its widespread use, treatment response remains variable with as few as $30 \%$ of patients achieving a $50 \%$ or more reduction in seizure frequency. ${ }^{8,37}$ Although VNS has been used in clinical practice for more than two decades, the exact mechanistic underpinnings of its effect in modulating seizures remain poorly understood and patient-related predictive factors of treatment response have yet to be established.

Initial investigation into the mechanisms of VNS largely focused on individual anatomical structures and neurotransmitter systems within the brain. However, in recent years, advances in brain imaging and computational tools have enabled large-scale mapping of neural networks across various brain regions. Application of connectomicsbased approaches to the field of epilepsy has begun to shed light on the complex network between brainstem centers and subcortical and cortical regions that may underlie the antiseizure effects of VNS. In this review we outline current data on the underlying neural circuitry believed to be implicated in VNS responsiveness in what we term the "vagus afferent network" and explore current evidence for the use of VNS in pediatric epilepsy. We further discuss the emerging role of biomarkers to predict treatment effect and highlight important avenues of future work. Ultimately, uncovering the constellation of neural circuits that mediates VNS response may help better inform patient selection and guide stimulation parameters to optimize treatment effect.

\section{Methods of Investigating Vagal Afferent Circuitry}

Initial studies examining the anatomical structures implicated in VNS response relied on protein detection meth-

ABBREVIATIONS BDNF = brain-derived neurotrophic factor; BOLD = blood oxygenation level dependent; DRN = dorsal raphe nucleus; EEG = electroencephalography; fEPSP = field excitatory postsynaptic potential; FGF = fibroblast growth factor; fMRI = functional MRI; GABA = gamma-aminobutyric acid; IL = interleukin; LC = locus coeruleus; NTS = nucleus tractus solitarius; PBN = parabrachial nucleus; SSEP = somatosensory evoked potential; VNS = vagus nerve stimulation.

SUBMITTED April 29, 2018. ACCEPTED June 15, 2018.

INCLUDE WHEN CITING DOI: 10.3171/2018.6.FOCUS18216. 
ods and reporter enzyme tracing to uncover vagal afferent circuitry and elucidate regions of neuronal activation. Some of the earliest studies on the vagal afferent network utilized horseradish peroxidase retrograde cell labeling to define connections between brainstem nuclei and subcortical structures downstream of vagal afferent signaling. The target neuroanatomical structures involved in VNS response were later determined by immunohistochemical analyses of neuronal activation markers such as cFos. ${ }^{53}$ Moreover, application of microdialysis techniques in animal models of epilepsy treated with VNS demonstrated significant changes in neurotransmitter levels within key brain regions, thus further defining a network of functional circuitry activated by VNS. ${ }^{57}$

The introduction of advanced electrophysiological tools and functional imaging techniques has revolutionized our ability to investigate neural networks in both spatial and temporal dimensions. Many studies to date have utilized surface electrode readings to examine changes in evoked potentials following VNS and ultimately determine potential biomarkers of response. ${ }^{52}$ Application of BOLD functional MRI (fMRI) and PET imaging in clinical studies has revealed structural networks that are activated by VNS in humans and further demonstrated dynamic changes in functional circuitry that occur over time..$^{15,34,44}$ More recently, resting-state fMRI and phase- and amplitude-based studies of oscillatory connectivity have provided insights into the interrelations among key nodes of the vagus afferent network. As the field of neural connectomics continues to grow, advancements in multimodal neuroimaging will likely increase the capability of further investigating VNS afferent pathways.

\section{The Vagus Afferent Network}

Approximately $80 \%$ of vagus nerve fibers are afferent sensory fibers that relay both somatic and general visceral signals. Vagal afferents primarily project to the nucleus tractus solitarius (NTS), which in turn sends fibers to other brainstem nuclei important in modulating the activity of subcortical and cortical circuitry (Fig. 1). Together this "vagus afferent network" is likely the neural substrate of VNS-mediated antiseizure effects, and further study into its complex circuitry will help elucidate predictors of treatment response.

\section{Brainstem Centers}

Attempts to map the underlying neural networks involved in VNS response has led to the identification of critical brainstem nuclei and neurotransmitter systems that appear to be early mediators in the pathway of seizure modulation.

\section{Nucleus Tractus Solitarius}

The NTS serves as a major relay center of vagal afferent projections. It receives direct inputs from the vagus nerve and subsequently projects to other brainstem nuclei including the locus coeruleus (LC), dorsal raphe nucleus (DRN), and parabrachial nucleus (PBN), along with forebrain limbic structures such as the paraventricular nucleus of the hypothalamus, stria terminalis, and cingulate cortex..$^{59} \mathrm{VNS}$ has been shown to increase c-Fos expression, a marker of neuronal activity, within the NTS along with its downstream targets during both acute and chronic treatment paradigms..$^{18}$ The functional importance of NTS connectivity in modulating seizure activity is further borne out by findings that increased inhibitory gamma-aminobutyric acid (GABA) signaling or decreased excitatory glutamate signaling within the NST reduces susceptibility to chemically induced limbic motor seizures. ${ }^{65}$

\section{Locus Coeruleus}

Noradrenergic signaling is an important mediator of VNS antiseizure effect. The LC, the primary site of noradrenergic neurons within the brain, receives direct inputs from the NTS and projects widely to limbic structures. Multiple lines of evidence have implicated the LC in mediating the VNS effect and sustaining long-term alterations in functional activity. Short bursts of VNS increase neuronal firing in the LC within minutes, leading to elevations in norepinephrine concentrations within the amygdala, hippocampus, and prefrontal cortex..$^{25,28,33,60}$ This increase in norepinephrine has been correlated with positive treatment response..$^{57}$ Moreover, stimulation of the LC has been shown to suppress epileptic activity within the amygdala, ${ }^{35}$ and LC lesions block VNS antiepileptic effects. ${ }^{38}$ Chronic VNS has also been shown to induce long-lasting activation of LC neurons. ${ }^{23}$

\section{Dorsal Raphe Nucleus}

VNS has been shown to regulate serotonergic pathways in the brain through modulation of the DRN within the brainstem. Whether the DRN receives direct inputs from the NTS has been an area of debate. However, electrophysiological studies have demonstrated indirect projections by way of the LC to the DRN, which, in turn, sends widespread projections to upper cortical regions. Unlike the LC, the DRN appears to have a more delayed response to VNS. Acute VNS does not lead to changes in DRN neuronal activity. ${ }^{18}$ However, application of VNS for at least 14 days increased neuronal firing within the DRN and chronic treatment maintained long-term enhancement of baseline activity. ${ }^{23}$ Importantly, this effect was abolished when the LC was lesioned, thus supporting an LC-DRN synaptic pathway of effect. Moreover, selective destruction of serotonergic neurons inhibited the antiepileptic effects of VNS in a chemically induced seizure model..$^{14}$

\section{Parabrachial Nucleus}

Vagal afferents project to the PBN in the dorsolateral pontine tegmentum by way of both the NTS and LC. Cell bodies within the PBN send diffuse outputs to forebrain structures including the thalamus, insular cortex, amygdala, and hypothalamus. ${ }^{54}$ Blood oxygenation level-dependent (BOLD) fMRI analysis of patients with epilepsy receiving VNS has revealed activation in downstream PBN targets including frontal lobes, insula, and cingulate cortex.$^{44}$ Moreover, PBN likely plays an important role in regulating thalamocortical circuitry that may be implicated in seizure generation. Specifically, PBN activates the intralaminar nuclei of the thalamus, which in turn relays sensory signals to widespread cortical areas. Motelow et al. found decreased activity of intralaminar thalamic nuclei and cholinergic neurotransmission in focal temporal 


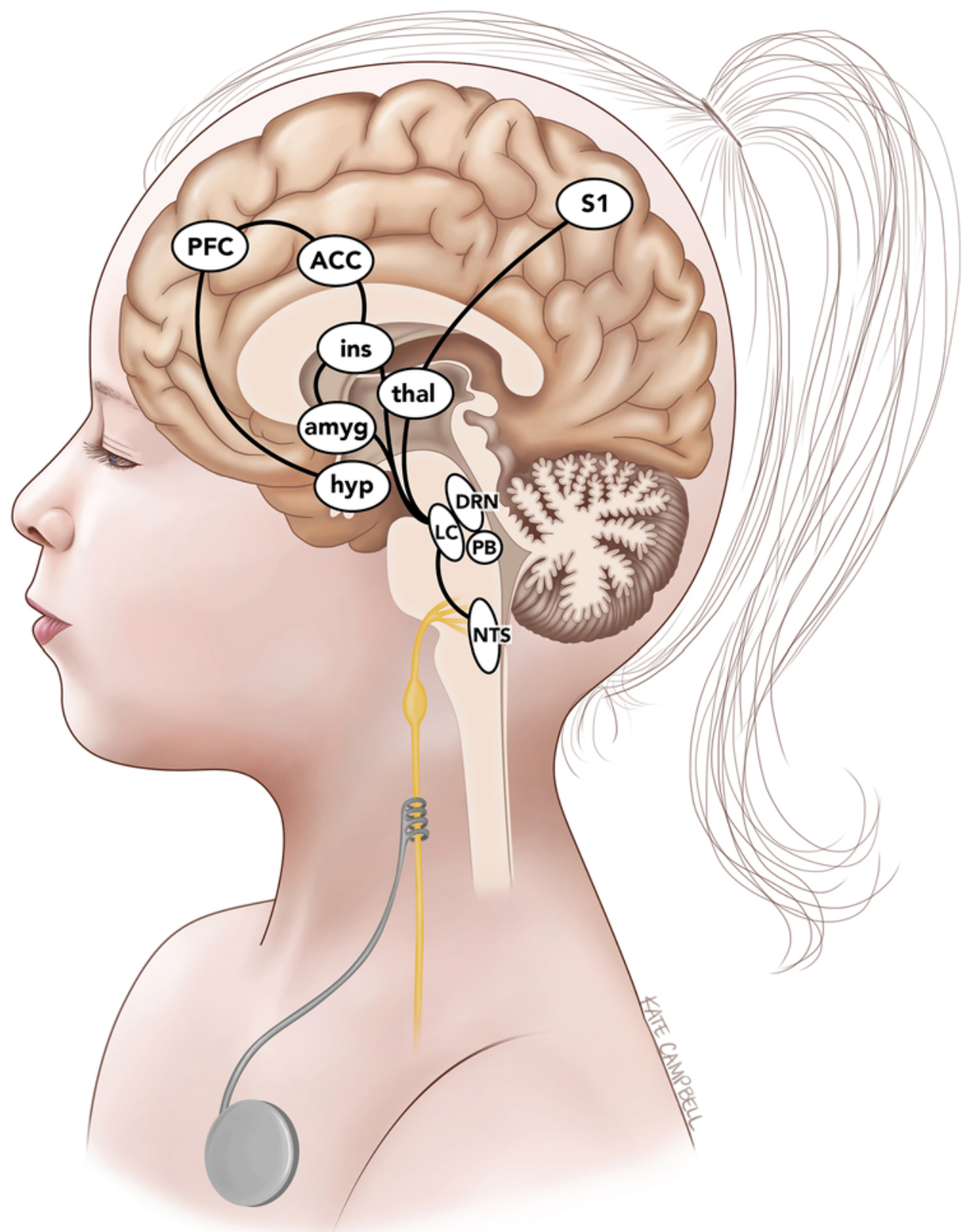

FIG. 1. The vagus afferent network. Schematic diagram showing the important brainstem centers and subcortical and cortical structures that likely underlie VNS treatment effect. $\mathrm{ACC}=$ anterior cingulate cortex; amyg = amygdala; hyp = hypothalamus; ins = insula; $\mathrm{PB}=$ parabrachial nucleus; $\mathrm{PFC}$ = prefrontal cortex; S1 = primary somatosensory cortex; thal = thalamus. Copyright Kate Campbell, Medical \& Scientific Visualizations. Published with permission.

lobe epilepsy. ${ }^{49}$ This suggests a potential role for PBN and its upstream cholinergic inputs in mediating the VNS antiepileptic effect.

\section{Subcortical and Cortical Circuitry}

Early studies into the mechanism of action of VNS revealed widespread involvement of various subcortical and cortical structures.$^{53}$ With the introduction of cortical mapping techniques and advanced functional imaging, recent work has begun to elucidate the underlying circuitry between these structures along with the temporal changes in connectivity induced by VNS (Table 1).

\section{Thalamocortical Connections}

Alterations in structural and functional thalamocortical circuitry have long been implicated in seizure generation and propagation. As such, thalamocortical connections are believed to be an important substrate of VNS responsiveness. The thalamus receives direct inputs from the NTS through the central tegmental tract and also receives ascending input from the PBN and noradrenergic pathways by way of the LC. ${ }^{7}$ Early studies demonstrated changes in regional cerebral blood flow and neuronal activation within the thalamus following VNS..$^{32}$ In addition, chronic VNS has been shown to increase thalamocortical somatosenso- 
ry evoked potential (SSEP) latency, a phenomenon that has been associated with antiepileptic drug treatment. ${ }^{52}$ More recently, thalamic activation measured by BOLD fMRI was associated with improved VNS treatment response in patients with seizures. ${ }^{44}$ The importance of thalamocortical connections in mediating the VNS antiseizure effect is further borne out by findings that thalamic connections to the anterior cingulate and insular cortices are stronger in VNS responders. ${ }^{34}$

\section{Plasticity Within Amygdala and Hippocampal Circuits}

Multiple lines of work have implicated plasticity within the amygdala and hippocampus as important contributors to VNS response. Expression changes within the postsynaptic density proteome of the amygdala and piriform cortex have been documented following 1 week of VNS stimulation. ${ }^{3}$ Specifically, expression of neurexin-1a, cadherin 13 , and a2d1 proteins were upregulated, all of which are important drivers of excitatory synapse formation. Moreover, VNS has been shown to increase hippocampal gene expression of brain-derived neurotrophic factor (BDNF) and fibroblast growth factor (FGF), ${ }^{25}$ and induce long-term cellular proliferation and neurogenesis within the hippocampus. ${ }^{11,58}$ It is postulated that these effects are modulated by noradrenergic and serotonergic imputs. ${ }^{39,47}$ How increased neuronal plasticity may mediate VNS treatment effects remains unclear. Hippocampal atrophy has been implicated in the pathogenesis of temporal lobe epilepsy and as a predictor of seizure onset. ${ }^{27}$ Moreover, aberrant circuitry within the hippocampus due to both abnormal axonal sprouting and loss of regulation of inhibitory inputs has been linked to seizure generation. ${ }^{2}$ Increased neurotrophic factor expression and neurogenesis within these brain regions may therefore provide a mechanism to form new synapses and rewire dysregulated circuitry. ${ }^{11}$

There is growing evidence to suggest that VNS alters neuronal excitability within the amygdala and hippocampus. VNS has been shown to increase seizure threshold of neurons in the amygdala. ${ }^{4}$ Moreover, VNS decreases field excitatory postsynaptic potential (fEPSP) slope and electroencephalography (EEG) power as well as slows theta rhythms within the hippocampus, thus suggesting a potential effect in decreasing excitability and subsequently seizure generation..$^{42,43}$ It is postulated that this effect may, in part, be mediated by noradrenergic signaling to presynaptic $\alpha 2 \mathrm{~A}$-adrenoceptors within the dentate gyrus, which blocks presynaptic release of excitatory neurotransmitters. ${ }^{10}$

\section{Reorganization of Limbic Circuitry}

Recent work has also suggested that VNS may reorganize functional circuits within the limbic system. Using BOLD fMRI data in rats receiving VNS, Cao et al. found increased functional connectivity between the retrosplenial cortex and hippocampus along with connectivity between the sensory cortex and striatum, ${ }^{15}$ circuits presumed to mediate memory and learning along with sensorimotor integration and cognition, respectively. ${ }^{6,63}$ In contrast, VNS decreased connectivity between the cingulate cortex and ventral striatum, suggesting a decrease in emotional processing. While this alteration in functional circuitry may, in part, explain the antidepressive effects observed with VNS, how these changes modulate seizure threshold remains unclear. Nevertheless, these findings underscore the importance of conducting further large-scale functional and temporal analyses of cortical circuitry alterations following VNS.

\section{Immunomodulation by Way of Hypothalamic Regulation}

There is emerging evidence to suggest that VNS evokes an immunomodulatory response that may contribute to its antiepileptic effect. The vagus nerve is intimately associated with the hypothalamic-pituitary-adrenal axis. Vagal afferents by way of the NTS project to corticotrophin-releasing factor neurons in the parvocellular paraventricular nucleus of the hypothalamus, which in turn leads to activation of the adrenocorticotropic hormone-glucocorticoid pathway, ultimately exerting an antiinflammatory effect. ${ }^{13}$ Increased serum corticosterone levels have been demonstrated in rodents 1 hour after VNS. ${ }^{20}$ Moreover, chronic VNS in patients with refractory epilepsy led to a significant decrease in the proinflammatory cytokine interleukin (IL)$8 .{ }^{19}$ Majoie et al. found decreased peripheral blood levels of the proinflammatory cytokine IL-6 and increased levels of the antiinflammatory cytokine IL-10 in responders to VNS. The opposite was observed in VNS nonresponders. ${ }^{46}$ Whether these observations reflect a direct modulatory effect of VNS on inflammatory centers or a biomarker of responsiveness remains unclear. However, with increasing lines of evidence suggesting that inflammation may be an important factor in seizure generation and propagation, ${ }^{62}$ future studies are warranted to better characterize the potential immunomodulatory effects of VNS.

\section{Cerebellar Circuitry}

Compared to thalamic and limbic connections, considerably less is known about the contribution of cerebellar circuitry to VNS responsiveness in epilepsy. Changes in cerebellar blood flow have been observed following VNS implantation, but the functional importance of this finding remains unclear. ${ }^{30}$ The cerebellum shows oscillatory neuronal activity during generalized seizures and cerebellar nuclei are known to send multiple projections to thalamocortical circuits. Further studies are necessary to better determine functional cerebellar pathways that may mediate VNS response.

\section{VNS for Pediatric Epileptic Syndromes}

Most of the early studies on the efficacy and safety of VNS for intractable epilepsy were conducted in adult cohorts. However, as this adjunct continues to gain widespread use, increasing evidence supports its utility in the setting of pediatric epilepsy syndromes.

\section{Efficacy Among Pediatric Patients}

One of the earliest studies focusing on VNS within the pediatric population examined 60 children with medically intractable epilepsy. ${ }^{50}$ Following 3 months of VNS treatment there was a $23 \%$ median reduction in seizure frequency across the cohort. At 18 months, median reduction in seizure frequency was $42 \%$ in 46 patients. Rychlicki et al. studied the effects of VNS in a cohort of 34 children 
TABLE 1. The vagus afferent network: summary of hypotheses

Summary

\begin{tabular}{cc}
\hline Brainstem center & $\begin{array}{c}\text { VNS increases c-Fos expression w/in NTS along w/ its downstream targets; increased inhibitory GABA } \\
\text { signaling or decreased excitatory glutamate signaling w/in the NST reduces susceptibility to chemically } \\
\text { induced limbic motor seizures }\end{array}$ \\
\hline LC & $\begin{array}{c}\text { Short bursts of VNS increase neuronal firing in LC leading to elevations in norepinephrine w/in the } \\
\text { amygdala, hippocampus, \& prefrontal cortex; increased norepinephrine is correlated with positive VNS } \\
\text { treatment response; stimulation of LC suppresses epileptic activity w/in the amygdala; LC lesions block } \\
\text { VNS antiepileptic effects }\end{array}$ \\
\hline VNS (for at least 14 days) increases neuronal firing w/in the DRN, which is abolished when the LC is \\
lesioned; selective destruction of serotonergic neurons inhibits VNS antiepileptic effects
\end{tabular}

with drug-resistant epilepsy. Mean reduction in total seizures was $39 \%$ by 3 months, $61 \%$ by 24 months, and $71 \%$ by 36 months. ${ }^{61}$ Patients with partial epilepsy had a better response than those with Lennox-Gastaut syndrome. No significant adverse side effects were found following implantation. Many other cohort studies have also shown that VNS is efficacious and well tolerated in pediatric patients with medically refractory epilepsy (summarized in Table 2). $., 9,12,29,64$

\section{Dravet Syndrome}

Dravet syndrome is a rare condition of childhood associated with intractable seizures. Few reports have examined the effect of VNS within this population; however, those that have suggest good treatment effect. In a meta-analysis including 68 patients with Dravet syndrome treated with adjunctive VNS, $53 \%$ of patients experienced $\geq 50 \%$ reduction in seizures. ${ }^{22}$ VNS was associated with a mean seizure reduction rate of $31 \%$ at 12 months in 8 pediatric patients with Dravet syndrome. ${ }^{67}$

\section{Lennox-Gastaut Syndrome}

Lennox-Gastaut syndrome is a childhood epileptogenic disorder associated with a range of different seizures types. If seizures are medically refractory, treatment consists of either VNS or corpus callosotomy. Lancman et al. found that corpus callosotomy had significantly improved outcomes compared to VNS for $>50 \%$ and $>70 \%$ atonic sei- zure reduction. However, other seizure types showed similar response rates between VNS and corpus callosotomy. ${ }^{41}$ In a multicenter retrospective study of pediatric patients with Lennox-Gastaut syndrome and medically refractory epilepsy, VNS was associated with a median reduction in total seizures of $42 \%$ at 1 month and $58 \%$ at 6 months. ${ }^{26}$

\section{Predictors of Response}

Given the heterogeneity in effectiveness of VNS among patients, there is a growing need to determine clinically relevant biomarkers to predict treatment response. Insight into the underlying antiepileptic mechanisms of action of VNS has helped uncover candidate patient and seizurerelated markers that can inform patient selection.

\section{Noninvasive Functional Connectivity and Electrical Recording}

Assessment of presurgical functional connectivity may offer a promising mechanism to predict VNS responders. Ibrahim et al. demonstrated that enhanced connectivity between the thalamus, anterior cingulate, and insular cortices is associated with improved response to VNS. ${ }^{34} \mathrm{In}$ addition, increased hippocampal norepinephrine levels have been positively associated with VNS-induced antiseizure efficacy. ${ }^{57}$ The P3 component of scalp-recorded event-related potentials has been used as a surrogate marker of LC activity. ${ }^{51}$ De Taeye et al. found that the P3 amplitude 
TABLE 2. Summary of selected studies investigating VNS for pediatric epilepsy

\begin{tabular}{|c|c|c|}
\hline Authors \& Year & Cohort & Findings \\
\hline $\begin{array}{l}\text { Benifla et al., } \\
2006\end{array}$ & 41 patients, mean age 13 yrs & $\begin{array}{l}15(38 \%) \text { had a seizure frequency reduction of }>90 \% \text { (mean follow-up } 31 \text { mos); } 15 \text { ( } 38 \%) \\
\text { failed to respond to VNS treatment; } 5 \text { required device removal secondary to side ef- } \\
\text { fects/complications; most common side effects were cough \& vocal disturbance }\end{array}$ \\
\hline $\begin{array}{l}\text { Helmers et al., } \\
2001\end{array}$ & $\begin{array}{l}125 \text { patients at baseline, } 95 \text { at } 3 \text { mos, } 56 \\
\text { at } 6 \text { mos, } 12 \text { at } 12 \text { mos; mean age at } \\
\text { implantation } 11.8 \text { yrs }\end{array}$ & $\begin{array}{l}\text { Average seizure reduction of } 36.1 \% \& 44.7 \% \text { at } 3 \& 6 \text { mos, respectively; side effects } \\
\text { included voice alteration \& coughing during stimulation }\end{array}$ \\
\hline $\begin{array}{l}\text { Blount et al., } \\
2006\end{array}$ & 6 patients $(<5$ yrs old $)$ & $\begin{array}{l}83 \% \text { of patients had a significant decrease in seizure frequency: } 2 \text { seizure-free }(33 \%), 3 \\
\text { improved }(50 \%), 1(17 \%) \text { no change in seizure status }\end{array}$ \\
\hline $\begin{array}{l}\text { Rychlicki et al., } \\
2006\end{array}$ & $\mathrm{n}=34$, mean age $11.5 \mathrm{yrs}$ & $\begin{array}{l}\text { Mean reduction in total seizures was } 39 \% \text { by } 3 \text { mos, } 61 \% \text { by } 24 \text { mos, \& } 71 \% \text { by } 36 \text { mos; } \\
\text { side effects included voice alteration \& coughing during stimulation }\end{array}$ \\
\hline $\begin{array}{l}\text { Wakai \& Kota- } \\
\text { gal, } 2001\end{array}$ & $\begin{array}{l}5 \text { patients, median age at implantation } \\
12.7 \mathrm{yrs}\end{array}$ & $\begin{array}{l}\text { Seizure frequency was reduced }>50 \% \text { after VNS in } 4 / 5 \text { patients; no significant adverse } \\
\text { effects w/ VNS }\end{array}$ \\
\hline $\begin{array}{l}\text { Alexopoulos et } \\
\quad \text { al., } 2006\end{array}$ & $\begin{array}{l}46 \text { patients, median age at implantation } \\
12.1 \mathrm{yrs}\end{array}$ & $\begin{array}{l}\text { Median seizure-frequency reduction was } 56 \% \text { ( } 3 \text { mos), } 50 \% \text { ( } 6 \text { mos), } 63 \% \text { ( } 12 \text { mos), } 83 \% \\
\text { ( } 24 \text { mos), \& } 74 \% \text { ( } 36 \text { mos); } 20 \text { patients }(43.5 \% \text { ) had }>75 \% \text { seizure-frequency reduction; } \\
19 \text { patients ( } 41.3 \% \text { ) had no response; } 21.7 \% \text { of patients discontinued VNS due to lack } \\
\text { of efficacy or infection }\end{array}$ \\
\hline Murphy, 1999 & 60 patients, median age $15 \mathrm{yrs}$ & $\begin{array}{l}\text { After } 3 \text { mos of VNS treatment, there was a median reduction of } 23 \% \text { in seizure frequency; } \\
\text { at } 18 \text { mos, median reduction in seizure frequency was } 42 \% \text { in } 46 \text { patients }\end{array}$ \\
\hline
\end{tabular}

was significantly increased in VNS responders compared to nonresponders. Noninvasive scalp recording characteristics may therefore hold promise as potential noninvasive biomarkers to predict treatment response. ${ }^{21}$

\section{Seizure Semiology}

Seizure characteristics may also lend insight into VNS responsiveness. Casazza et al. found that patients with temporal region seizure onset had the greatest response to VNS compared to those with ictal frontal, central, or diffuse discharges. However, this study was significantly limited by a small sample size. ${ }^{16}$ In a meta-analysis of 74 clinical studies including 3321 patients undergoing VNS for epilepsy, it was found that generalized epilepsy was associated with greater benefit from VNS compared to partial seziures. ${ }^{24}$ Moreover, it was found that patients with posttraumatic epilepsy or tuberous sclerosis had better seizure control with VNS than patients with unknown or idiopathic etiology. ${ }^{24}$ Within a cohort of 58 pediatric patients, Kim et al. found that children with focal or multifocal epileptiform discharges on EEG were more likely to respond to VNS compared to those with generalized epileptiform activities. $^{36}$

\section{Patient Factors}

While still in its infancy, there is growing research into patient-related predictive factors to predict VNS responsiveness. Some reports have suggested that shorter duration of epilepsy prior to VNS may predict a favorable outcome. Moreover, patient age at the time of VNS implantation has been suggested as a potential predictor of response with children and adolescents showing improved outcomes compared to adults in some cohorts. ${ }^{17}$ There has yet to be any strong evidence to suggest sex-related differences in responsiveness.

\section{Insights Into Other Disease Processes}

In recent years, increased understanding of the vagus afferent network has enabled the application of VNS to a variety of neuropsychiatric conditions including depression, ${ }^{1}$ schizophrenia, ${ }^{56}$ dementia ${ }^{48}$ and obesity. ${ }^{55}$ Early insight into the potential effect of VNS on emotional regulation stemmed from studies of VNS in epileptic patients who noted improvements in mood following treatment. PET imaging studies have revealed decreased activity of structures implicated in mood regulation following VNS such as the amygdala, hippocampus, and cingulate cortex. ${ }^{31}$ Moreover, the known effect of VNS on noradrenergic and serotonergic pathways, neurotransmitter systems that play a key role in depression, ${ }^{23}$ suggests an underlying mechanism of action for mood regulation.

The finding that VNS reduces hippocampal hyperactivity has suggested a potential role for this treatment in the management of cognitive dysfunction in the setting of schizophrenia. Hyperactivity within the ventral hippocampus has previously been linked to abnormal dopamine signaling, which underlies cognitive disturbances associated with schizophrenia. ${ }^{45}$ Treatment with VNS was subsequently shown to reduce hippocampal activity and aberrant dopamine signaling, resulting in attenuation of positive symptoms in an animal model of schizophrenia. ${ }^{56}$ Ultimately, further understanding of the neural circuits within the vagus afferent network may enable application of VNS therapy to a multitude of other neuropsychiatric conditions.

\section{Conclusions}

VNS has gained widespread use for the treatment of refractory epilepsy. The emergence of functional connectomics and the introduction of large-scale modeling of neural networks has helped elucidate the underlying circuitry 
within the vagus afferent network that may be involved in VNS treatment effect and responsiveness. Further work in defining epileptogenic networks and understanding the regional and temporal circuit changes induced by VNS will be paramount in better informing appropriate patient selection and optimizing stimulation parameters.

\section{References}

1. Aaronson ST, Sears P, Ruvuna F, Bunker M, Conway CR, Dougherty DD, et al: A 5-year observational study of patients with treatment-resistant depression treated with vagus nerve stimulation or treatment as usual: comparison of response, remission, and suicidality. Am J Psychiatry 174:640-648, 2017

2. Alexander A, Maroso M, Soltesz I: Organization and control of epileptic circuits in temporal lobe epilepsy. Prog Brain Res 226:127-154, 2016

3. Alexander GM, Huang YZ, Soderblom EJ, He XP, Moseley MA, McNamara JO: Vagal nerve stimulation modifies neuronal activity and the proteome of excitatory synapses of amygdala/piriform cortex. J Neurochem 140:629-644, 2017

4. Alexander GM, McNamara JO: Vagus nerve stimulation elevates seizure threshold in the kindling model. Epilepsia 53:2043-2052, 2012

5. Alexopoulos AV, Kotagal P, Loddenkemper T, Hammel J, Bingaman WE: Long-term results with vagus nerve stimulation in children with pharmacoresistant epilepsy. Seizure 15:491-503, 2006

6. Balleine BW, Delgado MR, Hikosaka O: The role of the dorsal striatum in reward and decision-making. J Neurosci 27:8161-8165, 2007

7. Beckstead RM, Morse JR, Norgren R: The nucleus of the solitary tract in the monkey: projections to the thalamus and brain stem nuclei. J Comp Neurol 190:259-282, 1980

8. Ben-Menachem E, Mañon-Espaillat R, Ristanovic R, Wilder BJ, Stefan H, Mirza W, et al: Vagus nerve stimulation for treatment of partial seizures: 1. A controlled study of effect on seizures. Epilepsia 35:616-626, 1994

9. Benifla M, Rutka JT, Logan W, Donner EJ: Vagal nerve stimulation for refractory epilepsy in children: indications and experience at the Hospital for Sick Children. Childs Nerv Syst 22:1018-1026, 2006

10. Bickler PE, Hansen BM: Alpha 2-adrenergic agonists reduce glutamate release and glutamate receptor-mediated calcium changes in hippocampal slices during hypoxia. Neuropharmacology 35:679-687, 1996

11. Biggio F, Gorini G, Utzeri C, Olla P, Marrosu F, Mocchetti I, et al: Chronic vagus nerve stimulation induces neuronal plasticity in the rat hippocampus. Int $\mathbf{J}$ Neuropsychopharmacol 12:1209-1221, 2009

12. Blount JP, Tubbs RS, Kankirawatana P, Kiel S, Knowlton R, Grabb PA, et al: Vagus nerve stimulation in children less than 5 years old. Childs Nerv Syst 22:1167-1169, 2006

13. Bonaz B, Sinniger V, Pellissier S: Anti-inflammatory properties of the vagus nerve: potential therapeutic implications of vagus nerve stimulation. J Physiol 594:5781-5790, 2016

14. Browning RA, Clark KB, Naritoku DK, Smith DC, Jensen RA: Loss of anticonvulsant effect of vagus nerve stimulation in the pentylenetetrazol seizure model following treatment with 6-hydroxydopamine or 5,7-dihydroxytryptamine. Soc Neurosci Abstr 23:2424, 1997

15. Cao J, Lu KH, Powley TL, Liu Z: Vagal nerve stimulation triggers widespread responses and alters large-scale functional connectivity in the rat brain. PLoS One 12:e0189518, 2017

16. Casazza M, Avanzini G, Ferroli P, Villani F, Broggi G: Vagal nerve stimulation: relationship between outcome and electroclinical seizure pattern. Seizure 15:198-207, 2006
17. Colicchio G, Policicchio D, Barbati G, Cesaroni E, Fuggetta F, Meglio M, et al: Vagal nerve stimulation for drug-resistant epilepsies in different age, aetiology and duration. Childs Nerv Syst 26:811-819, 2010

18. Cunningham JT, Mifflin SW, Gould GG, Frazer A: Induction of c-Fos and DeltaFosB immunoreactivity in rat brain by vagal nerve stimulation. Neuropsychopharmacology 33:1884-1895, 2008

19. De Herdt V, Bogaert S, Bracke KR, Raedt R, De Vos M, Vonck K, et al: Effects of vagus nerve stimulation on proand anti-inflammatory cytokine induction in patients with refractory epilepsy. J Neuroimmunol 214:104-108, 2009

20. De Herdt V, Puimege L, De Waele J, Raedt R, Wyckhuys T, El Tahry R, et al: Increased rat serum corticosterone suggests immunomodulation by stimulation of the vagal nerve. J Neuroimmunol 212:102-105, 2009

21. De Taeye L, Vonck K, van Bochove M, Boon P, Van Roost D, Mollet L, et al: The P3 event-related potential is a biomarker for the efficacy of vagus nerve stimulation in patients with epilepsy. Neurotherapeutics 11:612-622, 2014

22. Dibué-Adjei M, Fischer I, Steiger HJ, Kamp MA: Efficacy of adjunctive vagus nerve stimulation in patients with Dravet syndrome: A meta-analysis of 68 patients. Seizure 50:147152,2017

23. Dorr AE, Debonnel G: Effect of vagus nerve stimulation on serotonergic and noradrenergic transmission. J Pharmacol Exp Ther 318:890-898, 2006

24. Englot DJ, Chang EF, Auguste KI: Vagus nerve stimulation for epilepsy: a meta-analysis of efficacy and predictors of response. J Neurosurg 115:1248-1255, 2011

25. Follesa P, Biggio F, Gorini G, Caria S, Talani G, Dazzi L, et al: Vagus nerve stimulation increases norepinephrine concentration and the gene expression of BDNF and bFGF in the rat brain. Brain Res 1179:28-34, 2007

26. Frost M, Gates J, Helmers SL, Wheless JW, Levisohn P, Tardo C, et al: Vagus nerve stimulation in children with refractory seizures associated with Lennox-Gastaut syndrome. Epilepsia 42:1148-1152, 2001

27. García-Fiñana M, Denby CE, Keller SS, Wieshmann UC, Roberts N: Degree of hippocampal atrophy is related to side of seizure onset in temporal lobe epilepsy. AJNR Am J Neuroradiol 27:1046-1052, 2006

28. Groves DA, Bowman EM, Brown VJ: Recordings from the rat locus coeruleus during acute vagal nerve stimulation in the anaesthetised rat. Neurosci Lett 379:174-179, 2005

29. Helmers SL, Wheless JW, Frost M, Gates J, Levisohn P, Tardo C, et al: Vagus nerve stimulation therapy in pediatric patients with refractory epilepsy: retrospective study. J Child Neurol 16:843-848, 2001

30. Henry TR, Bakay RA, Pennell PB, Epstein CM, Votaw JR: Brain blood-flow alterations induced by therapeutic vagus nerve stimulation in partial epilepsy: II. Prolonged effects at high and low levels of stimulation. Epilepsia 45:1064-1070, 2004

31. Henry TR, Bakay RA, Votaw JR, Pennell PB, Epstein CM, Faber TL, et al: Brain blood flow alterations induced by therapeutic vagus nerve stimulation in partial epilepsy: I. Acute effects at high and low levels of stimulation. Epilepsia 39:983-990, 1998

32. Henry TR, Votaw JR, Pennell PB, Epstein CM, Bakay RA, Faber TL, et al: Acute blood flow changes and efficacy of vagus nerve stimulation in partial epilepsy. Neurology 52:1166-1173, 1999

33. Hulsey DR, Riley JR, Loerwald KW, Rennaker RL II, Kilgard MP, Hays SA: Parametric characterization of neural activity in the locus coeruleus in response to vagus nerve stimulation. Exp Neurol 289:21-30, 2017

34. Ibrahim GM, Sharma P, Hyslop A, Guillen MR, Morgan BR, Wong S, et al: Presurgical thalamocortical connectivity is as- 
sociated with response to vagus nerve stimulation in children with intractable epilepsy. Neuroimage Clin 16:634-642, 2017

35. Jimenez-Rivera C, Voltura A, Weiss GK: Effect of locus ceruleus stimulation on the development of kindled seizures. Exp Neurol 95:13-20, 1987

36. Kim MJ, Yum MS, Kim EH, Lee YJ, Lee J, Hong S, et al: An interictal EEG can predict the outcome of vagus nerve stimulation therapy for children with intractable epilepsy. Childs Nerv Syst 33:145-151, 2017

37. Klinkenberg S, van den Bosch CN, Majoie HJ, Aalbers MW, Leenen L, Hendriksen J, et al: Behavioural and cognitive effects during vagus nerve stimulation in children with intractable epilepsy - a randomized controlled trial. Eur J Paediatr Neurol 17:82-90, 2013

38. Krahl SE, Clark KB, Smith DC, Browning RA: Locus coeruleus lesions suppress the seizure-attenuating effects of vagus nerve stimulation. Epilepsia 39:709-714, 1998

39. Kulkarni VA, Jha S, Vaidya VA: Depletion of norepinephrine decreases the proliferation, but does not influence the survival and differentiation, of granule cell progenitors in the adult rat hippocampus. Eur J Neurosci 16:2008-2012, 2002

40. Kwan P, Brodie MJ: Early identification of refractory epilepsy. N Engl J Med 342:314-319, 2000

41. Lancman G, Virk M, Shao H, Mazumdar M, Greenfield JP, Weinstein S, et al: Vagus nerve stimulation vs. corpus callosotomy in the treatment of Lennox-Gastaut syndrome: a meta-analysis. Seizure 22:3-8, 2013

42. Larsen LE, Wadman WJ, Marinazzo D, van Mierlo P, Delbeke J, Daelemans S, et al: Vagus nerve stimulation applied with a rapid cycle has more profound influence on hippocampal electrophysiology than a standard cycle. Neurotherapeutics 13:592-602, 2016

43. Larsen LE, Wadman WJ, van Mierlo P, Delbeke J, Grimonprez A, Van Nieuwenhuyse B, et al: Modulation of hippocampal activity by vagus nerve stimulation in freely moving rats. Brain Stimul 9:124-132, 2016

44. Liu WC, Mosier K, Kalnin AJ, Marks D: BOLD fMRI activation induced by vagus nerve stimulation in seizure patients. J Neurol Neurosurg Psychiatry 74:811-813, 2003

45. Lodge DJ, Grace AA: Aberrant hippocampal activity underlies the dopamine dysregulation in an animal model of schizophrenia. J Neurosci 27:11424-11430, 2007

46. Majoie HJ, Rijkers K, Berfelo MW, Hulsman JA, Myint A, Schwarz M, et al: Vagus nerve stimulation in refractory epilepsy: effects on pro- and anti-inflammatory cytokines in peripheral blood. Neuroimmunomodulation 18:52-56, 2011

47. Malberg JE, Eisch AJ, Nestler EJ, Duman RS: Chronic antidepressant treatment increases neurogenesis in adult rat hippocampus. J Neurosci 20:9104-9110, 2000

48. Merrill CA, Jonsson MA, Minthon L, Ejnell H, C-son Silander H, Blennow K, et al: Vagus nerve stimulation in patients with Alzheimer's disease: additional follow-up results of a pilot study through 1 year. J Clin Psychiatry 67:11711178,2006

49. Motelow JE, Li W, Zhan Q, Mishra AM, Sachdev RN, Liu $\mathrm{G}$, et al: Decreased subcortical cholinergic arousal in focal seizures. Neuron 85:561-572, 2015

50. Murphy JV: Left vagal nerve stimulation in children with medically refractory epilepsy. J Pediatr 134:563-566, 1999

51. Murphy PR, Robertson IH, Balsters JH, O'Connell RG: Pupillometry and P3 index the locus coeruleus-noradrenergic arousal function in humans. Psychophysiology 48:15321543,2011

52. Naritoku DK, Morales A, Pencek TL, Winkler D: Chronic vagus nerve stimulation increases the latency of the thalamocortical somatosensory evoked potential. Pacing Clin Electrophysiol 15:1572-1578, 1992

53. Naritoku DK, Terry WJ, Helfert RH: Regional induction of fos immunoreactivity in the brain by anticonvulsant stimulation of the vagus nerve. Epilepsy Res 22:53-62, 1995

54. Nemeroff CB, Mayberg HS, Krahl SE, McNamara J, Frazer A, Henry TR, et al: VNS therapy in treatment-resistant depression: clinical evidence and putative neurobiological mechanisms. Neuropsychopharmacology 31:1345-1355, 2006

55. Pelot NA, Grill WM: Effects of vagal neuromodulation on feeding behavior. Brain Res 1693 (Pt B):180-187, 2018

56. Perez SM, Carreno FR, Frazer A, Lodge DJ: Vagal nerve stimulation reverses aberrant dopamine system function in the methylazoxymethanol acetate rodent model of schizophrenia. J Neurosci 34:9261-9267, 2014

57. Raedt R, Clinckers R, Mollet L, Vonck K, El Tahry R, Wyckhuys T, et al: Increased hippocampal noradrenaline is a biomarker for efficacy of vagus nerve stimulation in a limbic seizure model. J Neurochem 117:461-469, 2011

58. Revesz D, Tjernstrom M, Ben-Menachem E, Thorlin T: Effects of vagus nerve stimulation on rat hippocampal progenitor proliferation. Exp Neurol 214:259-265, 2008

59. Ricardo JA, Koh ET: Anatomical evidence of direct projections from the nucleus of the solitary tract to the hypothalamus, amygdala, and other forebrain structures in the rat. Brain Res 153:1-26, 1978

60. Roosevelt RW, Smith DC, Clough RW, Jensen RA, Browning RA: Increased extracellular concentrations of norepinephrine in cortex and hippocampus following vagus nerve stimulation in the rat. Brain Res 1119:124-132, 2006

61. Rychlicki F, Zamponi N, Trignani R, Ricciuti RA, Iacoangeli M, Scerrati M: Vagus nerve stimulation: clinical experience in drug-resistant pediatric epileptic patients. Seizure 15:483490,2006

62. Vezzani A, French J, Bartfai T, Baram TZ: The role of inflammation in epilepsy. Nat Rev Neurol 7:31-40, 2011

63. Vogt BA, Finch DM, Olson CR: Functional heterogeneity in cingulate cortex: the anterior executive and posterior evaluative regions. Cereb Cortex 2:435-443, 1992

64. Wakai S, Kotagal P: Vagus nerve stimulation for children and adolescents with intractable epilepsies. Pediatr Int 43:61-65, 2001

65. Walker BR, Easton A, Gale K: Regulation of limbic motor seizures by GABA and glutamate transmission in nucleus tractus solitarius. Epilepsia 40:1051-1057, 1999

66. WHO: Atlas: Epilepsy Care in the World 2005. Geneva: World Health Organization, 2005

67. Zamponi N, Passamonti C, Cappanera S, Petrelli C: Clinical course of young patients with Dravet syndrome after vagal nerve stimulation. Eur J Paediatr Neurol 15:8-14, 2011

\section{Disclosures}

The authors report no conflict of interest concerning the materials or methods used in this study or the findings specified in this paper.

\section{Author Contributions}

Conception and design: Ibrahim, Hachem. Drafting the article: Ibrahim, Hachem. Critically revising the article: all authors. Reviewed submitted version of manuscript: all authors. Approved the final version of the manuscript on behalf of all authors: Ibrahim. Study supervision: Ibrahim.

\section{Correspondence}

George M. Ibrahim: University of Toronto, ON, Canada. george. ibrahim@sickkids.ca. 